\title{
Ultra-compact acousto-optic modulator of a fibre Bragg grating in a highly birefringent suspended core fibre
}

da Silva, Ricardo, Manuylovich, Egor, Sahoo, Namita, Becker, Martin, Rothhardt, Manfred, et al.

Ricardo E. da Silva, Egor Manuylovich, Namita Sahoo, Martin Becker, Manfred Rothhardt, Hartmut Bartelt, David J. Webb, "Ultra-compact acoustooptic modulator of a fibre Bragg grating in a highly birefringent suspended core fibre," Proc. SPIE 11773, Micro-structured and Specialty Optical Fibres VII, 117730 U (18 April 2021); doi: 10.1117/12.2592228 


\title{
Ultra-compact acousto-optic modulator of a fibre Bragg grating in a highly birefringent suspended core fibre
}

\author{
Ricardo E. da Silva ${ }^{\mathrm{a} *}$, Egor Manuylovich ${ }^{\mathrm{a}}$, Namita Sahoo ${ }^{\mathrm{a}}$, Martin Becker ${ }^{\mathrm{b}}$ \\ Manfred Rothhardt ${ }^{\mathrm{b}}$, Hartmut Bartelt ${ }^{\mathrm{b}}$ and David J. Webb ${ }^{\mathrm{a}}$ \\ ${ }^{a}$ Aston Institute of Photonic Technologies, Aston University, Birmingham, B4 7ET, UK \\ ${ }^{b}$ Leibniz Institute of Photonic Technology, Jena, 07745, Germany
}

\begin{abstract}
Highly efficient and compact acousto-optic modulator of a fibre Bragg grating is reported for the first time. The device is composed of millimetre scaled components and a $1 \mathrm{~cm}$ grating inscribed in a four air holes birefringent suspended core fibre. The reflection of the orthogonal polarization modes is tuned by a sinusoidal electrical signal at the resonance frequency of $469 \mathrm{kHz}$. A significant modulation depth of $45 \%$ is achieved at a maximum drive voltage of $10 \mathrm{~V}$. The demonstrated $4 \mathrm{~cm}$ long all-fibre modulator is $60 \%$ shorter compared to previous similar setups, indicating new possibilities for stable and fast switching of fibre-integrated photonic devices.
\end{abstract}

Keywords: acousto-optic modulator, fibre Bragg grating, birefringent suspended core fibre.

\section{INTRODUCTION}

Fibre Bragg gratings (FBGs) inscribed into birefringent optical fibres have potential applications in multiwavelength reflection filters, fibre sensors and fibre lasers ${ }^{1-6}$. These devices usually employ polarization controllers or cascaded cavities with single or few-mode high birefringent fibres to generate laser outputs from one up to six-wavelengths ${ }^{5-7}$. However, just by using a conventional polarization controller does not provide dynamic and fast tuning of the wavelength channels, since such devices are usually limited to change the grating reflection properties. Furthermore, the combination of cascaded cavities or many gratings may induce high insertion losses, increasing the number of components and the device size. In contrast, laser output properties, such as, power, wavelength, pulse width and repetition rate are electrically tuned by the frequency and power of all-fibre acousto-optic modulators. In particular, the interaction of fibre Bragg gratings (FBGs) and longitudinal acoustic waves has been successfully employed in multiwavelength tuneable filters, Q-switched and mode-locked fibre lasers ${ }^{8-13}$. A coaxial acousto-optic device is basically composed of a piezoelectric transducer (PZT), an acoustic silica horn and an optical fibre segment axially aligned. The PZT generates the acoustic waves being coupled along the fibre with the silica horn. Fig. 1(a) illustrates the reflection spectrum of a non-modulated FBG of period $\Lambda$ and reflectivity $r(\lambda)$. The acoustically induced strain $S_{\mathrm{z}}$ along the fibre core changes the grating period $\Lambda$ and the modal effective index $n_{\text {eff, }}$, modulating reflection lobes on both sides of the Bragg wavelength $\lambda_{B}=2 n_{\text {eff }} \Lambda$ (Fig. 1(b)). The modulated reflectivity $\eta$ and separation $\Delta \lambda$ of the $n^{\text {th }}$ order reflection lobe is given as ${ }^{14,15}$,

$$
\begin{gathered}
\eta=\tanh ^{2}\left[\frac{\pi \Delta n_{a c} \Gamma}{\lambda_{B}} L_{g} J_{m}\left(\frac{\lambda_{a}}{\Lambda} S_{z \max }\right)\right], \\
S_{z \max }=\sqrt{\frac{2 P_{a c}}{Y A_{s} v_{e x t}}}, \\
\Delta \lambda=\frac{f \lambda_{B}{ }^{2}}{2 n_{e f f} v_{e x t}},
\end{gathered}
$$

*r.da-silva@aston.ac.uk; phone 0121204 5311;

Micro-structured and Specialty Optical Fibres VII, edited by Kyriacos Kalli, Alexis Mendez, Pavel Peterka, Proc. of SPIE Vol. 11773, 117730U · (c) 2021 SPIE · CCC code: 0277-786X/21/\$21 · doi: 10.1117/12.2592228 
which depends on the grating and fibre properties, such as modulation amplitude of the refractive index $\Delta n_{a c}$, grating period $\Lambda$ and length $L_{g}$, modal confinement factor $\Gamma$, Young's modulus $Y$ and fibre cross section $A_{s}$. Considering that the acoustic velocity $v_{e x t}$ depends on the fibre material, the modulated grating reflectivity $\eta$ and separation $\Delta \lambda$ are basically tuned by the acoustic power $P_{a c}$ and frequency $f$ (period $\lambda_{\mathrm{a}}$ ), respectively. $J_{n}$ is $n^{\text {th }}$ order Bessel function of the first kind. The increasing power $P_{a c}$ decreases the grating reflectivity at the Bragg wavelength $\lambda_{B}$ by coupling the reflected power to higher-order side lobes. Acoustic frequencies in general lower than $600 \mathrm{kHz}$ induce a small separation $\Delta \lambda$ between the lobes, overlapping as the modulated envelope illustrated in Fig. 1(c) ${ }^{16}$.

An optical fibre with linear birefringence supports two polarization modes propagating with distinct effective indices and phase velocities. The "fast $\mathrm{Y}$ " mode propagates with a higher velocity $v_{\mathrm{Y}}=c / n_{\text {eff }}$, compared to the "slow $\mathrm{X}$ " mode velocity $v_{\mathrm{X}}=c / n_{\text {effX }}$. The interaction of the orthogonal modes with a grating results in two reflected bands centred at $\lambda_{\mathrm{Y}}$ and $\lambda \mathrm{x}$. Accordingly, the acoustically induced strain (Fig. 1(d)) modulates reflection lobes on both sides of the fast and slow modes (Fig. 1(e)), broadening the grating spectrum as illustrated in Fig. 1(f). However, in a standard single-mode fibre (SMF), the acoustic power is distributed over the whole fibre cross section, with just a small fraction overlapping with the grating. The reduction of the fibre cross section $A_{s}$ employing cladding-etched and tapered techniques is useful to strengthen the acousto-optic interaction ${ }^{10,14,17}$. High modulation efficiencies have been achieved by increasing the modulation index $\Delta n_{\mathrm{ac}}$ and acoustic power $P_{\mathrm{ac}}{ }^{17,18}$, or by employing long FBGs. Devices employing grating lengths from $L_{g}=5-12 \mathrm{~cm}$ have been reported ${ }^{12,19-21}$. However, fibre tapering and etching techniques usually make such long devices mechanically fragile and exposed to surface contamination. In addition, the inscription of long gratings requires the use of long phase masks or equipment to shift the fibre and the phase mask with respect to the beam ${ }^{22}$. The use of long fibre or grating lengths increases the switching time of acousto-optic devices because the acoustic wave takes more time to travel along the fibre. Overall, besides the fibre/grating properties, the final operation efficiency and device size depend on the design and material of all components employed for acoustic generation (e.g., PZT, acoustic horn). Consequently, the device lengths are usually about $10 \mathrm{~cm}$ or longer. In this sense, suspended-core fibres (SCFs) are a promising option to enhance the overlapping between acoustic waves and FBGs in the fibre core ${ }^{23,24}$. The SCFs are generally composed of a very small core surrounded by large air holes which reduce the silica content over the fibre cross section $A_{s}$. High acousto-optic efficiencies are therefore achieved employing shorter fibre/grating lengths without reducing the fibre diameter ${ }^{23-25}$.

In this paper, we describe a special modulator design employing a highly birefringent suspended core fibre (HB-SCF) which significantly enhances the acousto-optic interaction and reduces the modulator size. In addition, an unexpected asymmetric modulation pattern of the orthogonal modes is shown for the first time, indicating new possibilities to adjust the polarization and equalize optical channels in fibre-based filters and fibre lasers.

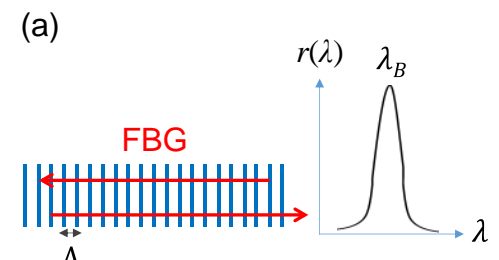

$\Lambda$ (b)

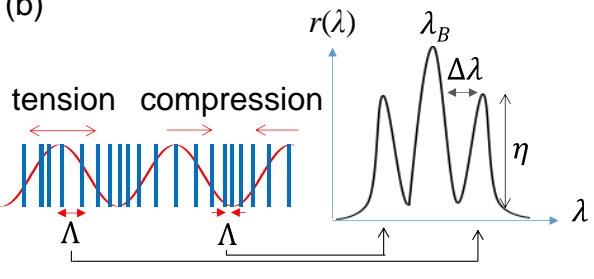

(c)

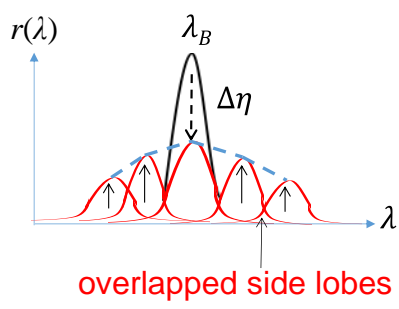

(d)

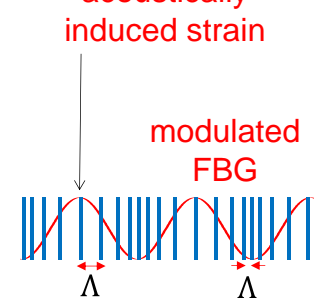

(e)

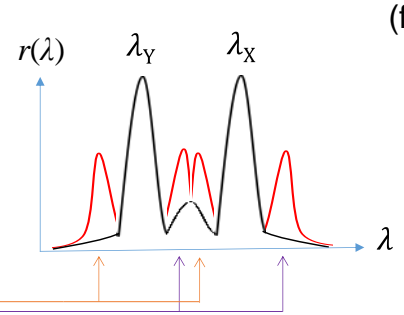

(f)

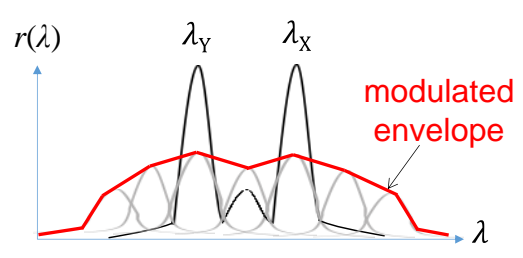

Figure 1. Illustration of a (a) non-modulated and an (b) acoustically modulated FBG spectrum for a SMF at (c) low frequencies. (d) The longitudinal acoustic strain (e) induces reflection bands on both sides of the orthogonal modes of a birefringent optical fibre, (f) which produce a modulated envelope in the grating spectrum. 


\section{EXPERIMENTAL SETUP}

Figure 2(a) shows the cross section of the highly birefringent double-core suspended-core fibre (HB-SCF) used in the experiment. The fibre is composed of a rectangular pure silica region $(14 \mu \mathrm{m} \times 7.7 \mu \mathrm{m})$ with a central air hole $(6 \mu \mathrm{m} \times$ $2 \mu \mathrm{m})$. The air hole forms two similar birefringent cores $(7.7 \mu \mathrm{m} \times 4 \mu \mathrm{m})$ surrounded by four air holes of $\sim 40-43 \mu \mathrm{m}$ in diameter, separated by silica bridges of $\sim 2.7 \mu \mathrm{m}$ thickness. The fibre diameter is $124 \mu \mathrm{m}$. A $1 \mathrm{~cm}$ long FBG is inscribed into the HB-SCF by means of a femtosecond laser (Coherent Mantis-Legend Elite-Tripler, $400 \mathrm{~mW}$ average power, $266 \mathrm{~nm}$ wavelength) and two-beam interference technique, using a phase mask interferometer according to the method described in Ref. ${ }^{22}$. The Bragg wavelength of the fast and slow modes are approximately $\lambda_{\mathrm{Y}}=1540.7 \mathrm{~nm}$ and $\lambda_{\mathrm{X}}=1540.9 \mathrm{~nm}$, respectively. The fibre birefringence is estimated from the reflection peaks as, $\Delta n_{\text {eff } \mathrm{XY}} \sim 2.2 \times 10^{-4}$.

Figure 2(b) illustrates the acousto-optic modulator composed of a piezoelectric transducer (PZT), a hollow silica horn and the HB-SCF with the inscribed grating. The piezo disc is $3 \mathrm{~mm}$ in diameter and $2 \mathrm{~mm}$ in thickness, as illustrated in Fig. 2(c). The acoustic horn is tapered along $\sim 1.4 \mathrm{~cm}$ in diameter from $\sim 1.06 \mathrm{~mm}$ to $\sim 200 \mu \mathrm{m}(\sim 150 \mu \mathrm{m}$ inner diameter at the tip). The horn is connected to the fibre and PZT by means of a glass adhesive, as indicated in Fig. 2(b). A conductive adhesive is used to fix the PZT to a metallic support and an electrode, making the electrical connection with an arbitrary signal generator (SG). This technique replaces the use of conventional soldering methods which cause undesired loads on the PZT. The SMF core is aligned only to one core of the HB-SCF, since the power coupling to the two SCF cores would induce high insertion losses. The fibres are spliced using a fusion splicer (Sumitomo F36).

The setup used in the experiment is illustrated in Fig. 2(d). The PZT is excited by a 1-10 V sinusoidal signal at the resonance of $f=469 \mathrm{kHz}$. The mechanical deformations of the transducer are amplified by the acoustic horn into longitudinal acoustic waves interacting with the grating. The modulated FBG spectrum is characterized by employing a coupler, a broadband source (LED Thorlabs ASE730) and an optical spectrum analyser (OSA Yokogawa AQ6370D) with a 20 pm wavelength resolution. The whole acousto-optic device is $4 \mathrm{~cm}$ long.

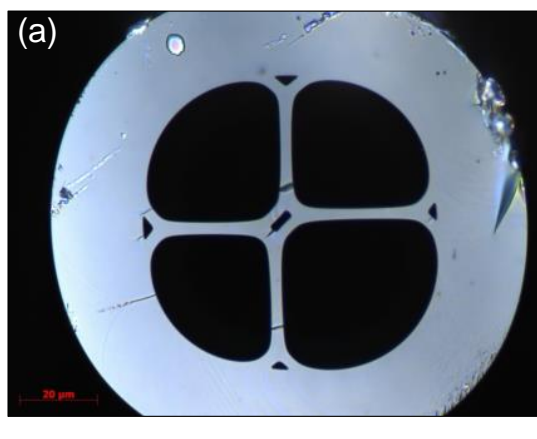

(c)
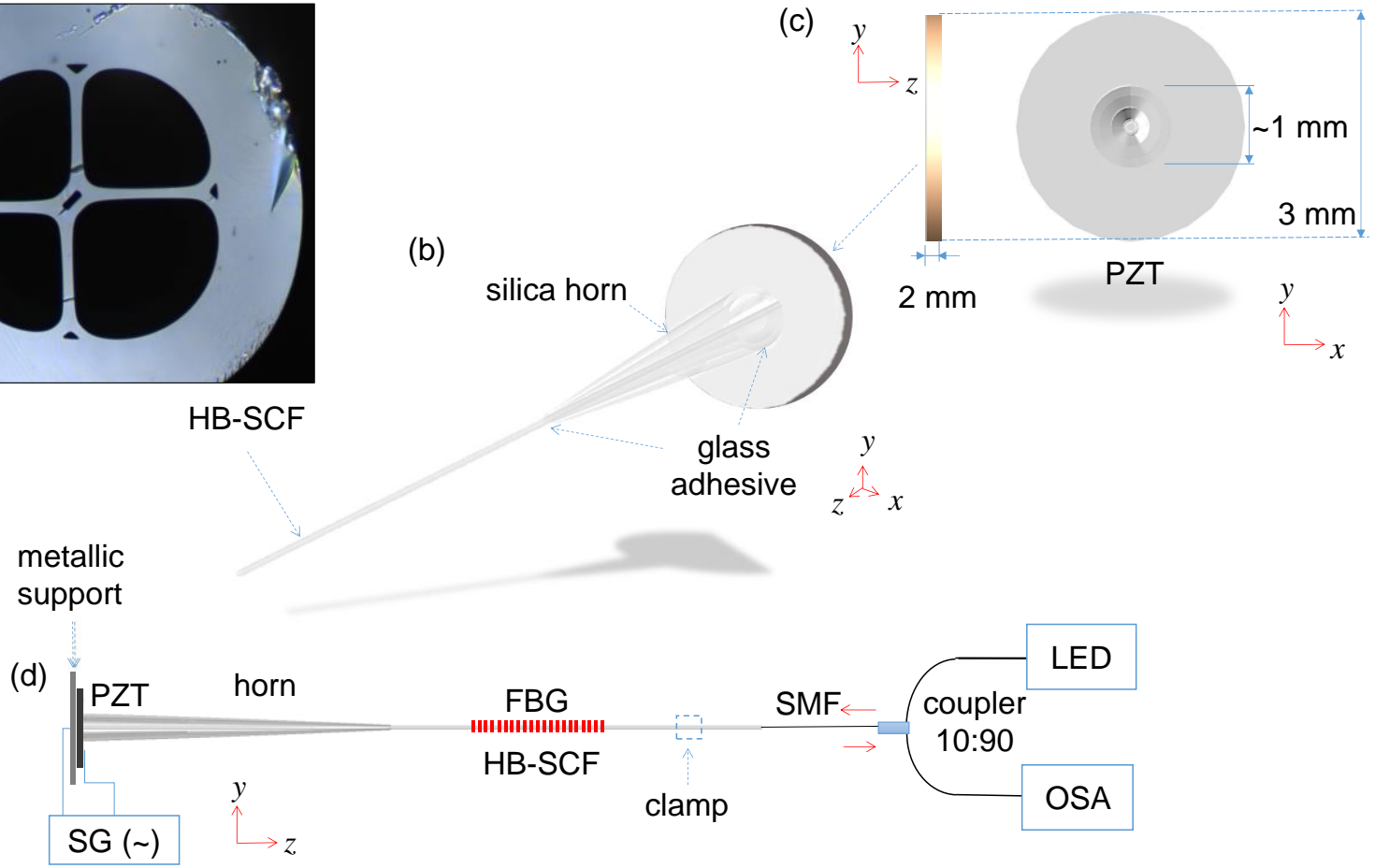

Figure 2. (a) Cross section image of the highly birefringent double-core suspended-core fibre (HB-SCF). (b) Illustration of the acousto-optic modulator with details of the (c) piezoelectric transducer (PZT). (d) Illustration of the experimental setup used to characterize the modulated FBG spectrum. 


\section{RESULTS AND DISCUSSION}

Figure 3 shows the non-modulated (voltage off) and the modulated FBG spectrum of the orthogonal modes represented by $\lambda_{\mathrm{Y}}$ and $\lambda_{\mathrm{X}}$ at the resonance of $f=469 \mathrm{kHz}$ and the $1-10 \mathrm{~V}$ voltage range applied to the PZT. The spectrum is normalized to the maximum reflectivity. The acoustic wave induces overlapped side lobes decreasing the reflectivity at the peaks. As expected, just the modulated envelope is seen. The peaks of the fast and slow modes achieve a maximum modulation depth of $\Delta \eta_{\mathrm{Y}} \sim 37 \%$ and $\Delta \eta_{\mathrm{X}} \sim 45 \%$ at $10 \mathrm{~V}$, respectively. Accordingly, the reflectivity difference between the peaks is reduced from $13 \%$ to $6 \%$. The modes show distinct modulated envelopes indicating an asymmetrical coupling from the peaks to the side lobes, as seen in Fig. 3.

The modulation depth $\Delta \eta$ for the considered piezo resonance and voltage range is shown in Fig. 4. The polarization peaks have similar $\Delta \eta$ response for the voltage range up to $4 \mathrm{~V}$ (difference of $\Delta \eta_{\mathrm{XY}}=\Delta \eta_{\mathrm{X}}-\Delta \eta_{\mathrm{Y}}=0.5 \%$ at $4 \mathrm{~V}$ ). This difference rises with increasing voltage and slight decreases at $7 \mathrm{~V}\left(\Delta \eta_{\mathrm{XY}}=2.5 \%\right)$, achieving a maximum of $\Delta \eta_{\mathrm{XY}}=7 \%$ at $10 \mathrm{~V}$. This unpredicted asymmetric pattern is qualitatively explained by considering individually each polarization mode in Eq. (1). The reflectivity discrepancy between the non-modulated peaks indicates a higher overlapping of optical power polarized to the slow mode $\lambda_{\mathrm{x}}$ interacting with the grating (confinement factor $\Gamma$ in Eq. 1). Consequently, the modulated reflectivity $\eta$ at $\lambda_{\mathrm{X}}$ is higher compared to the fast mode $\lambda_{\mathrm{Y}}$, reducing the initial discrepancy of the peaks. The coupling between the Bragg wavelength and the side lobes has a periodic nonlinear response with the acoustic power, which might induce slight oscillations in the modulation depth $\Delta \eta$ curve in Fig. $4^{10}$.

In summary, the demonstrated modulation pattern might be applied to tune dynamically the polarization between the orthogonal modes to $45^{\circ}$ and to equalize the power level of optical channels. The modulation depth is limited by the maximum $10 \mathrm{~V}$ of the employed signal generator. It is expected that higher voltages further increase the power coupled to the side lobes, additionally reducing the reflectivity difference between the peaks. The modulator design and the HBSCF contribute to a reduction of the fibre/grating length and the power applied to the PZT. The $4 \mathrm{~cm}$ long modulator is about up to $60 \%$ smaller compared to devices employing SMFs and HB-SCFs with lengths of about $10 \mathrm{~cm}$ operating in a near frequency range ${ }^{8,17,19,20,24}$. For the best of our knowledge, this is the smallest all-fibre coaxial acousto-optic device reported. Minor changes in the core geometry can adjust the fibre birefringence without significantly affecting the overall modulation efficiency.

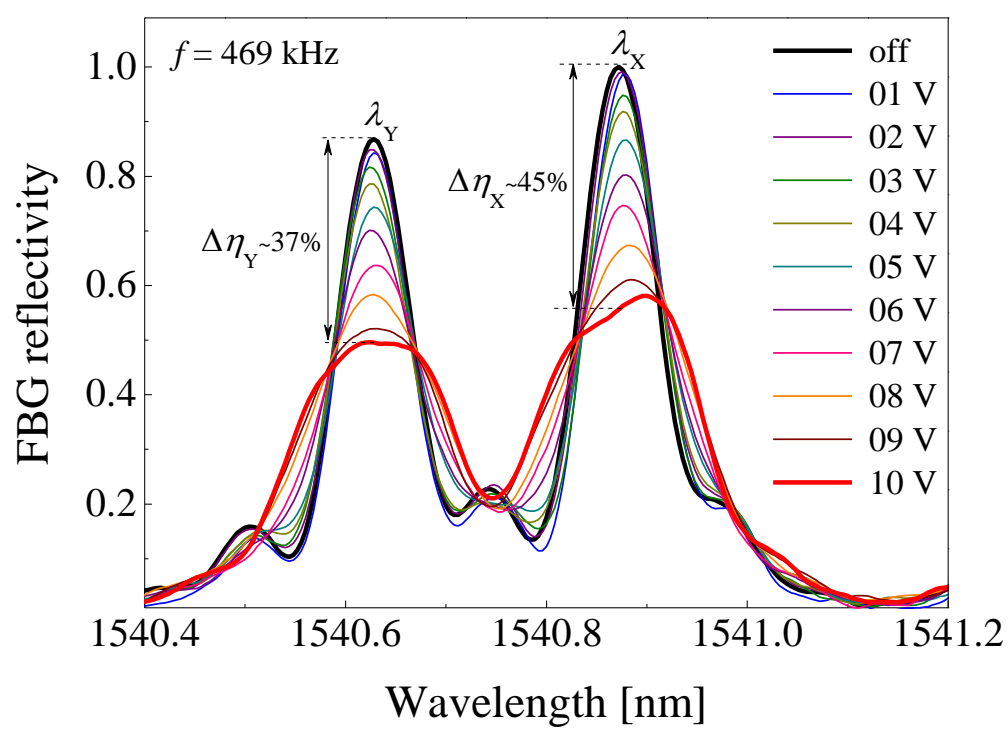

Figure 3. FBG spectra for the grating without and with acoustic modulation for the orthogonal polarization modes at the resonance frequency of $f=469 \mathrm{kHz}$. A voltage range of $1-10 \mathrm{~V}$ is applied to the PZT. 


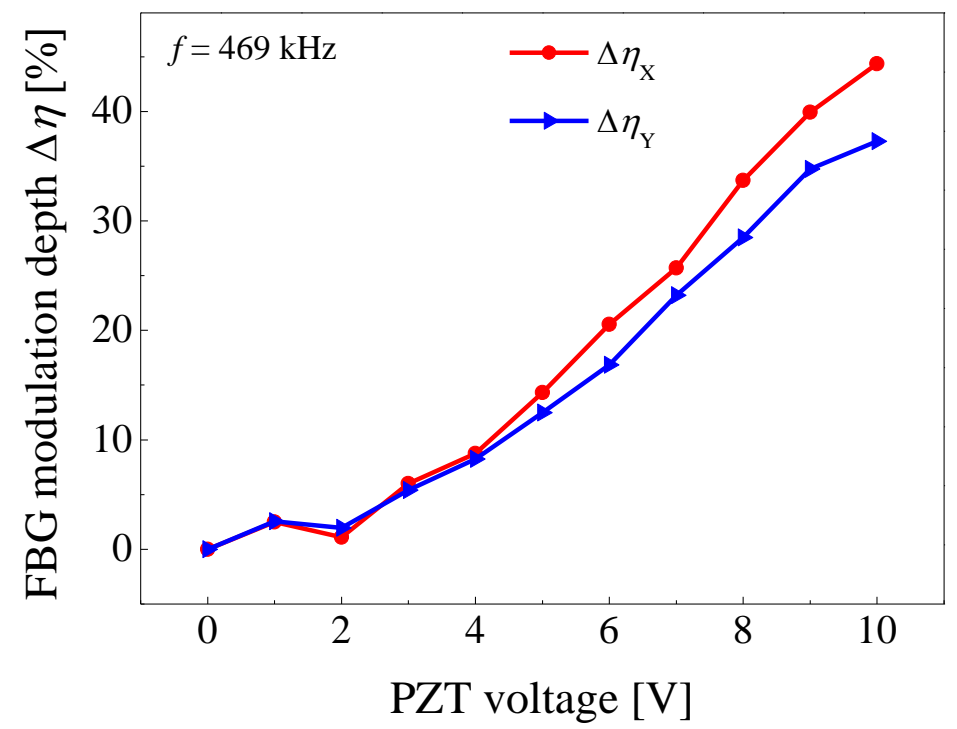

Figure 4. Modulation depth $\Delta \eta$ at the fast $\lambda_{\mathrm{Y}}$ and slow $\lambda \mathrm{x}$ polarization peaks at the resonance of $f=469 \mathrm{kHz}$ and the voltage range of $1-10 \mathrm{~V}$ applied to the PZT.

\section{CONCLUSION}

High efficiency acousto-optic modulation of $1 \mathrm{~cm}$ FBG in a highly birefringent suspended-core fibre is demonstrated in a compact $4 \mathrm{~cm}$ long device for the first time. The reflectivity of the polarization peaks is tuned by the voltage of an electrical signal at the PZT resonance of $f=469 \mathrm{kHz}$. The peaks of the fast and slow modes achieve a maximum modulation depth of $\Delta \eta_{\mathrm{Y}} \sim 37 \%$ and $\Delta \eta_{\mathrm{X}} \sim 45 \%$ at $10 \mathrm{~V}$, respectively. The reflectivity difference between the peaks is reduced from $13 \%$ to $6 \%$. This unexpected modulation pattern might be useful to tune the polarization between the orthogonal modes to $45^{\circ}$ and to equalize the power level of optical channels. The new acousto-optic modulator is up to $60 \%$ smaller compared to devices employing SMFs and HB-SCFs, which is promising for the fast switching of compact all-fibre photonic devices.

\section{ACKNOWLEDGMENT}

This project has received funding from the European Union's Horizon 2020 research and innovation programme under the Marie Sklodowska-Curie grant agreement No 713694.

\section{REFERENCES}

[1] Zhang, W., Williams, J. A. R. and Bennion, I., "Polarization synthesized optical transversal filter employing high birefringence fiber gratings," IEEE Photonics Technol. Lett. 13(5) (2001).

[2] Chen, G., Liu, L., Jia, H., Yu, J., Xu, L. and Wang, W., "Simultaneous strain and temperature measurements with fiber Bragg grating written in novel Hi-Bi optical fiber," IEEE Photonics Technol. Lett. 16(1), 221-223 (2004).

[3] Geernaert, T., Luyckx, G., Voet, E., Nasilowski, T., Chah, K., Becker, M., Bartelt, H., Urbanczyk, W., Wojcik, J., De Waele, W., Degrieck, J., Terryn, H., Berghmans, F. and Thienpont, H., "Transversal load sensing with fiber bragg gratings in microstructured optical fibers,” IEEE Photonics Technol. Lett. 21(1), 6-8 (2009). 
[4] Leners, R., François, P. L. and Stéphan, G., "Simultaneous effects of gain and loss anisotropies on the thresholds of a bipolarization fiber laser," Opt. Lett. 19(4), 275 (1994).

[5] Hernandez-Cordero, J., Kozlov, V. A., Carter, A. L. G. and Morse, T. F., "Fiber laser polarization tuning using a Bragg grating in a Hi-Bi fiber," IEEE Photonics Technol. Lett. 10(7), 941-943 (1998).

[6] Liu, Y., Feng, X., Yuan, S., Kai, G. and Dong, X., "Simultaneous four-wavelength lasing oscillations in an erbium-doped fiber laser with two high birefringence fiber Bragg gratings," Opt. Express 12(10), 2056 (2004).

[7] Moon, D. S., Paek, U.-C. and Chung, Y., "Polarization controlled multi-wavelength Er-doped fiber laser using fiber Bragg grating written in few-mode side-hole fiber with an elliptical core," Opt. Express 13(14), 5574 (2005).

[8] Marques, C. A. F., Oliveira, R. A, Pohl, A. A. P., Canning, J. and Nogueira, R. N., "Dynamic control of a phaseshifted FBG through acousto-optic modulation," Opt. Commun. 284(5), 1228-1231 (2011).

[9] Pohl, A. A. P., Oliveira, R. A., Silva, R. E., Marques, C. A. F., Neves, P. de T., Cook, K., Canning, J. and Nogueira, R. N., "Advances and new applications using the acousto-optic effect in optical fibers," Photonic Sensors 3(1), 1-25 (2013).

[10] Delgado-Pinar, M., Zalvidea, D., Diez, A., Perez-Millan, P. and Andres, M., "Q-switching of an all-fiber laser by acousto-optic modulation of a fiber Bragg grating," Opt. Express 14(3), 1106-1112 (2006).

[11] Cuadrado-Laborde, C., Díez, A., Cruz, J. L. and Andrés, M. V., "Experimental study of an all-fiber laser actively mode-locked by standing-wave acousto-optic modulation," Appl. Phys. B 99(1-2), 95-99 (2009).

[12] Villegas, I. L., Cuadrado-Laborde, C., Abreu-Afonso, J., Díez, A., Cruz, J. L., Martínez-Gámez, M. A. and Andrés, M. V., "Mode-locked Yb-doped all-fiber laser based on in-fiber acoustooptic modulation," Laser Phys. Lett. 8(3), 227-231 (2011).

[13] Silva, R. E., Tiess, T., Becker, M., Eschrich, T., Rothhardt, M., Jäger, M., Pohl, A. A. P. and Bartelt, H., "Acousto-optic modulation of a fiber Bragg grating in suspended core fiber for mode-locked all-fiber lasers," Laser Phys. Lett. 12(4) (2015).

[14] Liu, W. F., Russell, P. S. J. and Dong, L., "100\% efficient narrow-band acoustooptic tunable reflector using fiber Bragg grating,” J. Light. Technol. 16(11), 2006-2009 (1998).

[15] Russell, P. S. J. and Liu, W.-F., “Acousto-optic superlattice modulation in fiber Bragg gratings,” J. Opt. Soc. Am. A 17(8), 1421 (2000).

[16] Silva, R. E., Franco, M. A. R., Neves, P. T., Bartelt, H. and Pohl, A. A. P., "Numerical and experimental analysis of the modulation of fiber Bragg gratings by low-frequency complex acoustic waves," Opt. Fiber Technol. 30, 17-22 (2016).

[17] Li, Z., Pei, L., Liu, C., Ning, T. and Yu, S., "Research on FBG-based longitudinal-acousto-optic modulator with Fourier mode coupling method," Appl. Opt. 51(30), 7314 (2012).

[18] Oliveira, R. A., Neves Jr., P. T., Pereira, J. T., Canning, J. and Pohl, A. A. P., "Vibration mode analysis of a silica horn-fiber Bragg grating device," Opt. Commun. 283(7), 1296-1302 (2010).

[19] Oliveira, R. A., Marques, C. A. F., Mayer, C. E. N., Pereira, J. T., Nogueira, R. N. and Pohl, A. A. P., "Single device for excitation of both flexural and longitudinal acousto-optic effects in fiber Bragg gratings," 2009 SBMO/IEEE MTT-S Int. Microw. Optoelectron. Conf., 546-549 (2009).

[20] Silva, R. E., Franco, M. A. R., Neves Jr., P., Bartelt, H. and Pohl, A. A. P., "Detailed analysis of the longitudinal acousto-optical resonances in a fiber Bragg modulator," Opt. Express 21(6), 6997-7007 (2013).

[21] Cuadrado-Laborde, C., Diez, A., Delgado-Pinar, M., Cruz, J. L. and Andrés, M. V., "Mode locking of an allfiber laser by acousto-optic superlattice modulation," Opt. Lett. 34(7), 1111 (2009).

[22] Becker, M., Bergmann, J., Brückner, S., Franke, M., Lindner, E., Rothhardt, M. W. and Bartelt, H., "Fiber Bragg grating inscription combining DUV sub-picosecond laser pulses and two-beam interferometry," Opt. Express 16(23), 19169 (2008).

[23] Silva, R. E., Hartung, A., Rothhardt, M., Pohl, A. A. P. and Bartelt, H., "Detailed numerical investigation of the interaction of longitudinal acoustic waves with fiber Bragg gratings in suspended-core fibers," Opt. Commun. 344, 43-50 (2015).

[24] Silva, R. E., Becker, M., Rothhardt, M., Bartelt, H. and Pohl, A. A. P., "Electrically tunable multiwavelength bragg grating filter acoustically induced in a highly birefringent suspended core fiber," IEEE Photonics J. 9(1) (2017).

[25] Silva, R. E., Becker, M., Hartung, A., Rothhardt, M., Pohl, A. A. P. and Bartelt, H., "Reflectivity and bandwidth modulation of fiber Bragg gratings in a suspended core fiber by tunable acoustic waves," IEEE Photonics J. 6(6), $1-8$ (2014). 